\title{
VIABILIDADE ECONÔMICA DO INVESTIMENTO EM PLATAFORMAS DE COLHEDORAS DE MILHO EM ESPAÇAMENTO REDUZIDO
}

\author{
VILNEI DE O. DIAS ${ }^{1}$, RENATO S. DE SOUZA ${ }^{2}$, AIRTON DOS S. ALONÇO ${ }^{3}$
}

\begin{abstract}
RESUMO: Com o objetivo de avaliar a relação custo/benefício da adoção de espaçamento reduzido na cultura do milho em relação à aquisição de uma plataforma específica para a colheita mecanizada de grãos, foi realizado um estudo considerando as diferentes variáveis que interferem no investimento. Para tal, foi confeccionado um fluxo de caixa diferencial. Foram calculados com base no fluxo de caixa os indicadores de viabilidade econômica Valor Presente Líquido (VPL), Período de Retorno do Capital (PRC) e Taxa Interna de Retorno (TIR). Fez-se, ainda, a análise de sensibilidade sobre o VPL para variações no aumento da produtividade. Os VPLs encontrados ficaram entre $\mathrm{R} \$-32.157,00$ e R \$ 484.022,00. O PRC variou de 1,74 a 17,13 anos, e a TIR de -0,70 a 55,04\%. A viabilidade depende, basicamente, do tamanho da área, nível tecnológico do produtor e ganhos de produtividade com a adoção do espaçamento reduzido.
\end{abstract}

PALAVRAS-CHAVE: custos de produção, colheita mecanizada, Zea mays.

\section{ECONOMICAL VIABILITY OF INVESTMENT CONCERNING TO PLATFORMS OF CORN HARVERSTER IN A REDUCED SPACE}

\begin{abstract}
With the objective of evaluating the cost/benefit relationship concerning to the adoption of reduced spacing in the culture of the corn in relation to the acquisition of a specific platform for the automated crop of grains, it was accomplished a study considering the different variables that interfere in the investment. For such, it was accomplished a differential cash flow. The calculation based in the cash flow the indicators of economical viability: Liquid Present Value (VPL), Payback (PRC) and Internal Tax of Return (TIR). It was made an Analysis of Sensibility on VPL for percentile variations in the increase of the productivity. Found VPL's were between US\$ $-16,575.00$ and US\$ 249,483.00. PRC varied from 1.74 to 17.13 years and TIR from -0.70 to $55.04 \%$. The viability depends on the size of the area, technological level of the producer and productivity earnings with adoption of the reduced spacing.
\end{abstract}

KEYWORDS: production costs, automated crop, Zea mays.

\section{INTRODUÇÃO}

O milho é uma das mais importantes culturas agrícolas do Rio Grande do Sul, sendo cultivado praticamente em todo o Estado, destacando-se nas áreas de pequena propriedade (SANGÓI et al., 2003). Não obstante a sua importância, essa cultura apresenta baixa produtividade, sendo a média do Estado de $3,8 \mathrm{t} \mathrm{ha}^{-1}$ (SECRETARIA DA COORDENAÇÃO E PLANEJAMENTO, 2005). Essas baixas produtividades em relação ao potencial produtivo da cultura levam à constante busca por alternativas que aumentem o seu rendimento. Uma dessas alternativas é a maximização do uso dos recursos pelas plantas com a escolha do melhor arranjo, ou seja, da sua melhor distribuição na área de cultivo (ARGENTA et al., 2001a), o que pode ser feito de três maneiras: variando a densidade de plantas, a distribuição das plantas na fileira de cultivo ou estreitando o espaçamento entre as mesmas (ARGENTA et al., 2001b).

\footnotetext{
${ }^{1}$ Acadêmico de Agronomia da UFSM, Santa Maria - RS, Fone: (0XX55) 8406.2085, vilneialuno@yahoo.com.br. Bolsista de Iniciação Científica PIBIC/CNPq.

${ }^{2}$ Eng $^{\mathrm{o}}$ Agrônomo, Prof. Dr., Departamento de Educação Agrícola e Extensão Rural, UFSM, Santa Maria - RS.

${ }^{3}$ Eng ${ }^{0}$ Agrícola, Prof. Dr., Departamento de Engenharia Rural, UFSM, Santa Maria - RS.

Recebido pelo Conselho Editorial em: 25-8-2006
}

Aprovado pelo Conselho Editorial em: 13-4-2007 
Porém, com a adoção de espaçamentos reduzidos entre linhas, o produtor pode ter problemas com diversas operações, principalmente na colheita mecanizada, devido às plataformas das colhedoras tradicionais serem projetadas para coletar plantas em espaçamentos convencionais (70 a $100 \mathrm{~cm}$ ). Atualmente, existem no mercado plataformas de colheita que permitem colher lavouras instaladas com espaçamentos reduzidos entre linhas; contudo, sua aquisição exige investimentos elevados (ALONÇO et al., 2006), o que precisa ser confrontado com os benefícios potenciais advindos da adoção dessa técnica. Os efeitos da redução do espaçamento entre linhas relatados na literatura são bastante heterogêneos. No Sul do Brasil, os incrementos obtidos na produtividade com a redução do espaçamento de $90-100 \mathrm{~cm}$ para $45-50 \mathrm{~cm}$ são de pequena magnitude, variando de zero a 10\% (REUNIÃO TÉCNICA ANUAL DE PESQUISA DE MILHO E SORGO, 2005). Diante disso, torna-se necessário fornecer aos produtores subsídios para a tomada de decisão, de investir ou não em uma nova tecnologia que, segundo OLIVEIRA \& MILAN (2001), só é incorporada pelo produtor com estímulos econômicos.

$\mathrm{Na}$ agricultura, o produtor necessita escolher quando e como realizar os procedimentos recomendados pela pesquisa, podendo, ainda, optar pela execução ou não de determinadas atividades. Muitas vezes, a decisão de aplicar as técnicas apropriadas ou recomendadas, visando a ganhos superiores, requer investimentos (FETT, 2000). O investimento, segundo GALESNE et al. (1999), consiste em uma empresa comprometer ou imobilizar capital, sob diversas formas, a fim de manter ou melhorar sua situação econômica. De acordo com os mesmos autores, a decisão de investir, invariavelmente, comporta riscos, os quais devem ser previstos quando da decisão de investir em determinado equipamento.

A seleção de uma máquina agrícola, bem como de um implemento, pode tornar-se uma tarefa árdua, pois a escolha do equipamento mais adequado para uma propriedade agrícola é uma das etapas mais importantes do processo produtivo, e há diversas variáveis que devem ser consideradas (BAIO et al., 2004). A aquisição do equipamento de colheita implica alto investimento e só é justificado se houver significativo efeito sobre a lucratividade da atividade. Para SOFFNER et al. (1993), a maximização da lucratividade do negócio agrícola é o que determina o gerenciamento ótimo da maquinaria agrícola e não o contrário, pois a simples minimização dos custos das máquinas não é condição suficiente para a maximização do lucro do sistema.

Assim, o objetivo deste trabalho foi avaliar a viabilidade econômica da aquisição de plataformas específicas para a colheita mecanizada de milho, bem como verificar em que condições a mesma se torna viável, de forma a subsidiar a tomada de decisão de produtores que estejam interessados em adotar essa técnica.

\section{MATERIAL E MÉTODOS}

O estudo foi realizado na Universidade Federal de Santa Maria (UFSM), pelo Núcleo de Ensaio de Máquinas Agrícolas (NEMA), com o apoio do Núcleo de Estudos e Pesquisas em Economia Agroindustrial (NEPEA), de onde partiram todas as ações referentes à coleta, organização e processamento dos dados analisados. Os dados referentes às plataformas de colheita para milho em espaçamento reduzido foram obtidos junto a três fábricas de plataformas; foram coletados preços e especificações técnicas dos diferentes modelos disponíveis no mercado. No que se refere ao aumento da produtividade da cultura do milho, com a adoção da técnica, foi realizada uma revisão bibliográfica na qual se verificou o ganho de produtividade alcançado em condições experimentais nas mais diferentes situações.

Após a determinação da capacidade operacional das colhedoras, de acordo com a metodologia proposta por MIALHE (1974), fez-se o dimensionamento da colhedora para os diferentes tamanhos de propriedades, variando de 100 a 400 ha. Considerou-se eficiência de campo de $75 \%$, velocidade de trabalho igual a $5,0 \mathrm{~km} \mathrm{~h}^{-1}$, jornada de trabalho diária de 10 horas e período de colheita sempre inferior a 22 dias, com o objetivo de evitar perdas por atraso na colheita de milho. Foi atribuído o valor mínimo de oito linhas $(4 \mathrm{~m})$ e máximo de 17 linhas por conjunto colhedora-plataforma, de acordo com os modelos disponíveis no mercado. Quando esse valor era 
ultrapassado, automaticamente passava-se à adoção de dois conjuntos com a metade do número de linhas, mantendo-se, assim, a mesma capacidade operacional, porém com duas colhedoras.

Partiu-se do princípio de que o agricultor hipotético já possuía a(s) colhedora(s) e que seu investimento foi apenas na plataforma de colheita, e suas despesas, operacionais. Não foi considerado o valor de possível venda de uma antiga plataforma de que o agricultor dispunha antes da adoção da nova tecnologia.

Além do tamanho da área, variou-se ainda a produtividade de 65 a 140 sacas $^{-1}{ }^{-1}(3.900$ a $8.400 \mathrm{~kg} \mathrm{ha}^{-1}$ ), enquadrando-se diferentes níveis de manejo da cultura do milho (SANGÓI et al., 2006). Considerou-se, ainda, a elevação da população de plantas em 10 mil plantas ha ${ }^{-1}$ para $^{2}$ cálculo de todos os indicadores, tomando-se o preço de $\mathrm{R} \$ 105,00$ por saca de 60 mil sementes. Todos os preços do estudo estão em reais e foram coletados em março de 2006 . O preço da saca de $60 \mathrm{~kg}$ de milho foi obtido de séries temporais do período de janeiro de 2002 a dezembro de 2005, considerando-se médias mensais do período atualizadas para dezembro de 2005.

Com base nos custos e benefícios de implantação do sistema, foi construído o fluxo de caixa diferencial com valores anuais para um horizonte de 10 anos, valor estimado da vida útil da plataforma. As entradas foram representadas pela diferença no Valor Bruto da produção em função de aumento na produtividade entre 4 e $8 \%$ para cálculo do VPL e de $5 \%$ para os demais indicadores, após a adoção do sistema, e pelo valor residual da plataforma de colheita aos 10 anos, igual a $15 \%$ do valor novo da mesma. As saídas se dividiram em despesas de investimento e despesas operacionais.

Neste caso específico em estudo, as despesas de investimento foram formadas apenas pelo valor inicial da plataforma, sem custos de financiamento. As despesas operacionais consideradas foram os gastos adicionais com sementes e manutenção da plataforma. Com base no fluxo de caixa diferencial, calcularam-se índices de avaliação do investimento, como Valor Presente Líquido (VPL), o qual pode ser definido como a soma algébrica dos valores descontados do fluxo de caixa a ele associado. Em outras palavras, é a diferença do valor presente das entradas menos o valor presente das saídas do Fluxo de Caixa (SILVA \& FONTES, 2005); ou, ainda, corresponde à concentração de todos os valores de um fluxo de caixa descontados para a data zero ou presente (KREUZ et al., 2005); Período de Retorno do Capital (PRC), o qual representa o tempo necessário para que os benefícios do projeto reponham o valor investido (KREUZ et al., 2005), ambos com taxa de desconto de $8 \%$ ao ano. Para cálculo do PRC, fez-se regressão linear entre o VPL acumulado e o horizonte do investimento de 10 anos. Após, igualou-se essa equação a zero, de modo a determinar o ponto em que os valores de Y (VPL acumulado em R\$) tocam o eixo X (horizonte, em anos); esses valores são o VPL de cada situação avaliada. Fez-se, ainda, análise de sensibilidade sobre o VPL, variando-se o incremento em produtividade de 4 a $8 \%$ somente para esse indicador, e determinou-se a Taxa Interna de Retorno do investimento (TIR) nas dimensões de 100 a 400 ha, com escalas de 50 ha nas produtividades mencionadas anteriormente com o ganho médio de 5\% acrescido sobre as quatro produtividades estudadas. A TIR, por definição, é a taxa de desconto que torna uma série de entradas e saídas equivalentes na data presente, ou seja, é o valor da taxa de desconto que torna o VPL igual a zero (BATALHA, 1997).

\section{RESULTADOS E DISCUSSÃO}

Na Tabela 1, são apresentados os valores de Período de Retorno do Capital (PRC) para diferentes áreas da lavoura de milho e produtividade em sacas $\mathrm{ha}^{-1}$, com desconto de $8 \%$ ao ano. $\mathrm{O}$

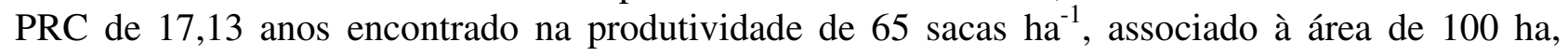
indica que o período necessário para que o capital investido retorne é superior ao horizonte do investimento considerado; neste caso, a vida útil estimada da plataforma, de 10 anos, de onde se deduz ser inviável o investimento nessa situação, segundo o indicador PRC. Verifica-se que o PRC diminui com o aumento da produtividade e da área, chegando a valores de 1,74 ano na produtividade de 140 sacas $\mathrm{ha}^{-1} \mathrm{em}$ área de 400 ha. Esses dados demonstram que existem economias de escala associadas a essa tecnologia de produção. 


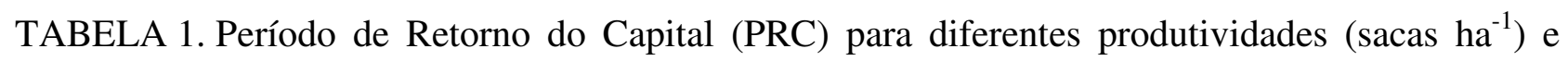
áreas de lavoura de milho (ha), considerado o aumento médio de $5 \%$ na produtividade.

\begin{tabular}{crrrr}
\hline \multirow{2}{*}{ Área (ha) } & \multicolumn{4}{c}{ Produtividade $\left(\right.$ sacas ha $\left.^{-1}\right)$} \\
\cline { 2 - 5 } & 65 & 90 & 115 \\
\cline { 2 - 4 } & \multicolumn{4}{c}{ PRC (anos) } \\
\hline 100 & 17,13 & 11,34 & 8,40 & 6,62 \\
150 & 11,54 & 7,50 & 5,48 & 4,27 \\
200 & 8,63 & 5,53 & 3,99 & 3,07 \\
250 & 6,84 & 4,33 & 3,08 & 2,34 \\
300 & 5,64 & 3,52 & 2,48 & 1,85 \\
350 & 5,35 & 3,33 & 2,33 & 1,74 \\
400 & 5,35 & 3,33 & 2,33 & 1,74 \\
\hline
\end{tabular}

Na Tabela 2, encontram-se as estimativas de Valor Presente Líquido (VPL), de acordo com aumentos percentuais em relação às produtividades de 65; 90; 115 e 140 sacas ha $^{-1}$ e tamanhos de lavoura de milho à taxa de desconto de $8 \%$ ao ano. Nota-se que, para aumento de $4 \%$ na produtividade de 65 sacas $^{-1} \mathrm{a}^{-1}$, áreas inferiores a 250 ha mostram-se inviáveis para o investimento. Com aumentos de 6\%, o investimento torna-se viável se forem cultivados 150 ha ou mais. Quando o aumento passa para $8 \%$, em áreas de 100 ha, o VPL já é positivo, o que indica a viabilidade do investimento. Entretanto, esse aumento de produtividade foi considerado teoricamente, sendo a média encontrada na literatura de 5\%. Considerando que propriedades menores têm, teoricamente, maior controle sobre seus fatores produtivos, esse aumento é plausível de ser alcançado, visto que, de acordo com REUNIÃO TÉCNICA ANUAL DE PESQUISA DE MILHO E SORGO (2005), esse valor tem sido demonstrado pela pesquisa variando de 0 a $10 \%$. Por outro lado, produtores maiores investem mais em tecnologia, tornando da mesma forma viável a obtenção de tal aumento da produtividade, principalmente em sistemas de manejo intensivo da cultura do milho. A afirmação anterior confere com os resultados de SANGÓI et al. (2006), os quais verificaram que o maior investimento em práticas de manejo e insumos incrementa o rendimento de grãos e a margem bruta da cultura do milho. Quando se aumenta a produtividade em 8\%, quanto maior o tamanho da área, maiores serão os rendimentos atualizados por meio do VPL, ou seja, as dimensões influenciam positivamente na rentabilidade do investimento, tornando o mesmo cada vez mais rentável.

Quando se eleva o nível tecnológico e, conseqüentemente, a produtividade da cultura para 90 sacas ha $^{-1}$, o investimento já se torna viável a partir de 200 ha, com VPL de R\$17.833,00 desde aumentos de $4 \%$ na produtividade. Esse VPL positivo indica viabilidade econômica do projeto de investimento, contudo deve ser olhado com atenção, pois esse valor dividido pelo número de anos e hectares apresentaria lucratividade baixa para o produtor. Já para a produtividade de 115 sacas ha $^{-1}$, o investimento passa a ser aprovado desde 100 ha, desde que se aumente $6 \%$ a produtividade com a adoção de espaçamento reduzido. Isso indica que, se o produtor de milho estiver adotando as demais práticas de manejo que garantam o bom desenvolvimento da cultura do milho, como adubação adequada, controle de pragas, plantas daninhas e doenças, uso da base genética mais adequada a cada situação (SANGÓI et al., 2006), esses níveis de aumento da produtividade são bastante acessíveis, considerando-se aí outros aspectos inerentes ao nível tecnológico da cultura (SANGÓI et al., 2003; FORSTHOFER \& SILVA, 2006). A produtividade é um elemento que está relativamente sob controle dos produtores, excetuando-se o aspecto clima (ESPERANCINI \& PAES, 2005).

Outro aspecto que deve viabilizar a aquisição da plataforma e insumos por pequenos produtores seria a compra em parceria com outros agricultores, como já é feito com diversos equipamentos, resguardada a complexidade de tal operação. 
TABELA 2. Análise de sensibilidade para o Valor Presente Líquido (VPL), de acordo com o tamanho da área (ha) e aumentos percentuais na produtividade (AP\%) sobre as produtividades de $65 ; 90 ; 115$ e 140 sacas $^{-1}$.

\begin{tabular}{|c|c|c|c|c|c|c|c|}
\hline \multirow{2}{*}{$\mathrm{AP}(\%)$} & \multicolumn{7}{|c|}{ Área (ha) } \\
\hline & 100 & 150 & 200 & 250 & 300 & 350 & 400 \\
\hline & \multicolumn{7}{|c|}{ VPL $(\mathrm{R} \$)$ na produtividade de 65 sacas $^{-1}{ }^{-1}$} \\
\hline 4 & -32.157 & -20.582 & -9.07 & 2.568 & 14.143 & 19.573 & 22.369 \\
\hline 6 & -14.711 & 5.587 & 25.885 & 46.183 & 66.481 & 80.634 & 92.153 \\
\hline \multirow[t]{2}{*}{8} & 2.736 & 31.757 & 60.778 & 87.799 & 118.820 & 141.696 & 161.938 \\
\hline & \multicolumn{7}{|c|}{ VPL $(\mathrm{R} \$)$ na produtividade de 90 sacas $\mathrm{ha}^{-1}$} \\
\hline 4 & -18.737 & -452 & 17.833 & 36.118 & 54.403 & 66.543 & 76.049 \\
\hline 6 & 5.420 & 35.783 & 66.146 & 96.509 & 126.872 & 151.090 & 172.674 \\
\hline \multirow[t]{2}{*}{8} & 29.576 & 72017 & 114.459 & 156.900 & 199.341 & 255.637 & 269.300 \\
\hline & \multicolumn{7}{|c|}{ VPL $(\mathrm{R} \$)$ na produtividade de $115 \mathrm{sacas} \mathrm{ha}^{-1}$} \\
\hline 4 & -5.316 & 19.679 & 44.674 & 69.669 & 94.664 & 113.514 & 129.730 \\
\hline 6 & 25.550 & 65.978 & 106.406 & 146.835 & 187.263 & 221.546 & 253.195 \\
\hline \multirow[t]{2}{*}{8} & 56.416 & 112.278 & 168.139 & 224.001 & 279.862 & 329.578 & 376.661 \\
\hline & \multicolumn{7}{|c|}{ VPL $(\mathrm{R} \$)$ na produtividade de 140 sacas $\mathrm{ha}^{-1}$} \\
\hline 4 & 8.104 & 39.809 & 71.514 & 103.219 & 134.924 & 160.484 & 493.411 \\
\hline 6 & 45.680 & 96.174 & 146.667 & 197.160 & 247.654 & 292.002 & 333.716 \\
\hline 8 & 83.257 & 152.538 & 221.820 & 291.101 & 360.383 & 423.519 & 484.022 \\
\hline
\end{tabular}

Na Tabela 3, são apresentados os valores da Taxa Interna de Retorno (TIR) para diferentes tamanhos da área de milho (ha), nas produtividades de 65;90;115 e 140 sacas ha ${ }^{-1}$. Se considerarmos a taxa de juros utilizada para o cálculo do VPL ( $8 \%$ ao ano), o investimento se tornaria rentável quando a TIR fosse superior a esse valor, ou seja, seria mais rentável o investimento do que simplesmente aplicar o recurso à taxa de desconto preestabelecida. Conforme se observa na Tabela 3, o investimento torna-se viável para as produtividades de $65 ; 90 ; 115$ e 140 sacas $^{-1}{ }^{-1}$ a partir das áreas de 200; 150; 100 e 100 ha, respectivamente. KREUZ et al. (2005), avaliando alternativas de investimento no Meio-Oeste Catarinense, consideraram uma taxa de desconto de $10 \%$. Se nesse caso fosse adotada tal taxa, o investimento seria atrativo nas mesmas condições. Da mesma forma, ESPERANCINI \& PAES (2005) analisaram o investimento na produção de café nos sistemas irrigado e convencional. Os autores atribuíram como taxa mínima de atratividade $12 \%$. Se considerarmos essa taxa para o investimento em questão, somente para a produtividade de 140 sacas $_{\text {ha }}{ }^{-1}$ o investimento seria viável considerando-se a TIR como indicador.

Observa-se que os valores da TIR evoluem à medida que aumenta a produtividade, sendo que os maiores incrementos ocorrem da produtividade de 65 para 90 sacas ha $^{-1}$. Quando se aumenta de

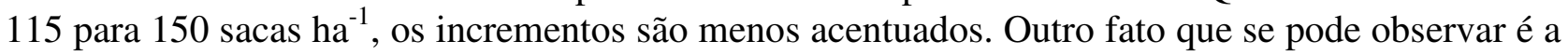
tendência à estabilização da TIR a partir dos 300 ha, o que sugere que, não havendo aumentos de rentabilidade do investimento a partir dessa área, ela deve ser o limite das economias de escala dessa tecnologia.

Na Figura 1, encontram-se os valores do VPL na área de 250 ha variando-se a taxa de desconto. Essas simulações tentam analisar o impacto da taxa de desconto sobre a viabilidade do investimento nessa dimensão de cultivo de milho tomada como exemplo. Verifica-se que, a partir da produtividade de 90 sacas ha $^{-1}$, acontece a diminuição do coeficiente angular da equação que explica a variação do VPL em função da taxa de desconto, significando que, na produtividade de 140 sacas $\mathrm{ha}^{-1}$, o impacto da taxa de desconto é menos drástico que na produtividade de 115 sacas ha ${ }^{-1}$ e, nessa, menos que em 90 sacas ha $^{-1}$. Isso ratifica que, em maiores produtividades, o investimento é mais rentável e, por isso, suporta maiores taxas de desconto. 
TABELA 3. Taxa Interna de Retorno (TIR) para variações no tamanho da área de lavoura de milho e quatro produtividades da lavoura, considerando $5 \%$ de aumento sobre as quatro produtividades estudadas.

\begin{tabular}{crrrr}
\hline \multirow{2}{*}{ Área (ha) } & \multicolumn{4}{c}{ Produtividades $\left(\mathrm{sacas} \mathrm{ha}^{-1}\right)$} \\
\cline { 2 - 5 } & \multicolumn{4}{c}{ TIR (\%) } \\
\cline { 2 - 5 } & $-0,70$ & 5,66 & 115 & 140 \\
\hline 100 & 5,40 & 13,79 & 21,40 & 16,65 \\
150 & 10,80 & 21,14 & 30,55 & 28,51 \\
200 & 15,88 & 28,05 & 39,35 & 39,56 \\
250 & 18,10 & 31,11 & 43,27 & 50,21 \\
300 & 18,10 & 31,12 & 43,28 & 55,03 \\
350 & 18,10 & 31,12 & 43,28 & 55,04 \\
400 & \multicolumn{4}{c}{5,04} \\
\hline
\end{tabular}
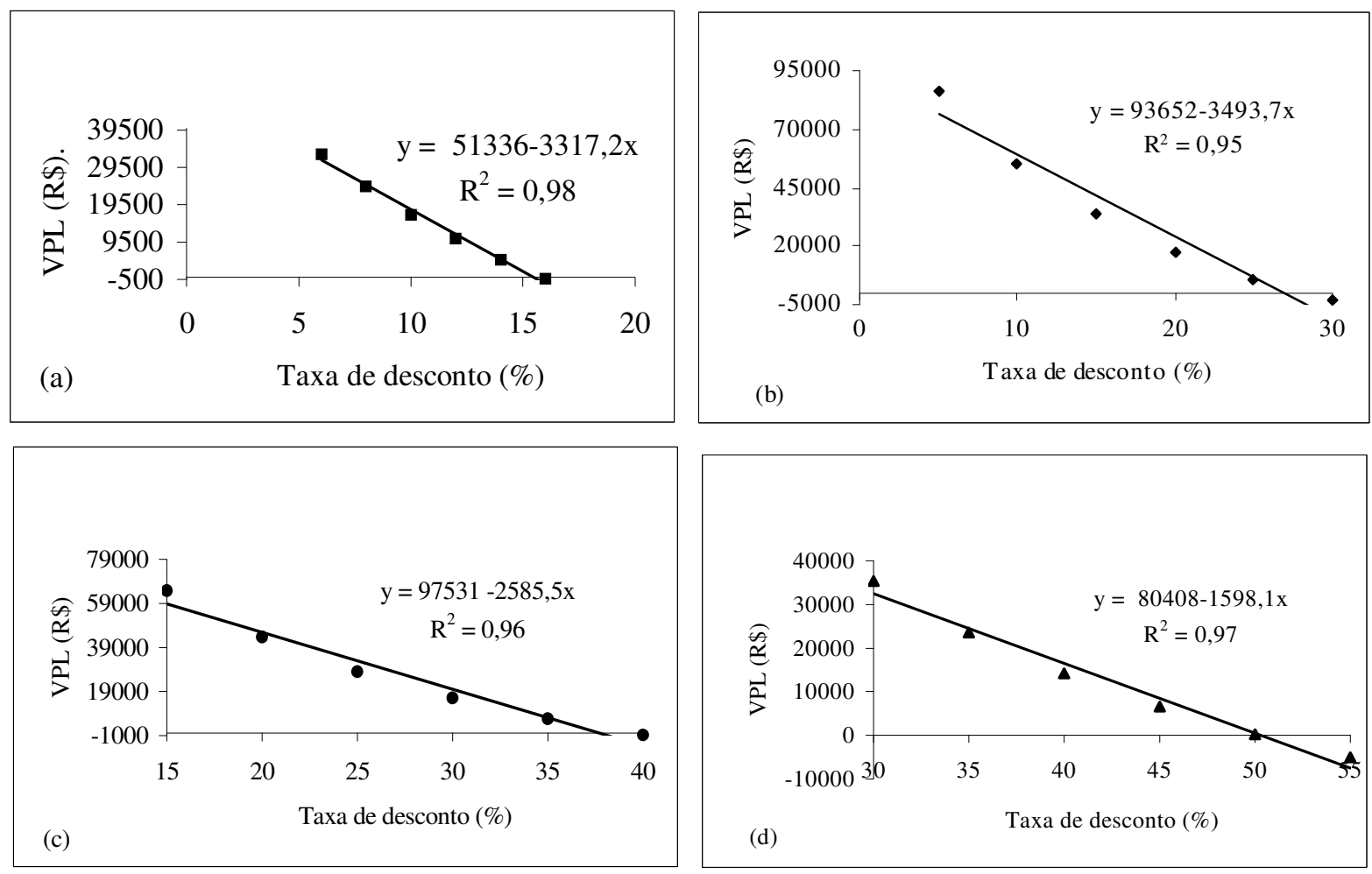

FIGURA 1. Evolução do Valor Presente Líquido (VPL) em função da taxa de desconto aplicada sobre a área de 250 ha, em produtividades de: (a) 65 sacas ha $^{-1}$, (b) 90 sacas ha $^{-1}$, (c) 115 sacas ha $^{-1}$ e (d) 140 sacas ha $^{-1}$.

Contudo, existem diversos aspectos, além dos financeiros, que devem ser avaliados pelo produtor antes que o mesmo resolva adotar a técnica de redução do espaçamento entre fileiras. Tais fatores podem ser apontados como "valores de opção" do investimento, isto é, vantagens potenciais que não podem ser computadas financeiramente, mas que são advindas do projeto de investimento. Segundo GALESNE et al. (1999), "valores de opção" são oportunidades futuras de investimento (das opções estratégicas decorrentes do investimento feito hoje, que passarão a fazer parte das opções estratégicas da empresa). Como exemplos, têm-se a diminuição da competição intraespecífica, pela melhor distribuição das plantas na área e, por conseqüência, aos aumentos do índice de área foliar da cultura, taxa de crescimento relativo e taxa de assimilação líquida (BULLOCK et al., 1988). Outra vantagem da adoção de espaçamentos reduzidos $(0,4 \mathrm{a} 0,5 \mathrm{~m})$ refere-se ao uso de semeadoras para milho, soja e feijão, sem que haja necessidade de mudanças de espaçamento entre linhas da semeadora, o que facilita o processo de semeadura dessas culturas, sem gasto de tempo para realizar as adaptações. Essa vantagem torna-se importante para 
agricultores que possuem apenas uma semeadora e necessitam semear milho, soja e feijão em período curto de tempo.

\section{CONCLUSÕES}

O produtor deve ser criterioso no momento de escolher reduzir o espaçamento entre fileiras na cultura do milho, pois a adoção dessa técnica implica despesas elevadas, o que, em determinadas situações, pode tornar o investimento economicamente inviável.

O investimento em plataformas de colheita pode ser viável em lavouras com produtividades de milho elevadas ou em grandes extensões de cultivo.

A viabilidade do investimento depende basicamente de três variáveis: escala de produção, o que indica existirem economias de escala associadas à tecnologia; produtividade da lavoura, e ganhos de produtividade com adoção da técnica, que estão relacionados ao nível de manejo empregado pelo produtor.

\section{REFERÊNCIAS}

ALONÇO, A. dos S.; DIAS, V. de O.; SOUZA, R.S. de; MEDEIROS, F.A.; MASSOCO, D. Viabilidade econômica da aquisição de plataforma de colheita para milho em espaçamento reduzido. In: CONGRESSO BRASILEIRO DE ENGENHARIA AGRÍCOLA, 36., 2006, João Pessoa. Anais... Jaboticabal: Associação Brasileira de Engenharia Agrícola, 2006. 1 CD-ROM.

ARGENTA, G.; SILVA, P.R.F. da; BORTOLINI, C.G.; FORSTHOFER, E.L.; MANJABOSCO, E.A.; NETO, V.B. Resposta de híbridos simples de milho à redução do espaçamento entre linhas. Pesquisa Agropecuária Brasileira, Brasília, v.36, n.1, p.71-8, 2001b.

ARGENTA, G.; SILVA, P.R.F. da; SANGÓI, L. Arranjo de plantas em milho: análise do estado da arte. Ciência Rural, Santa Maria, v.31, n.6, p.1075-84, 2001a.

BAIO, F.H.R.; ANTUNIASSI, U.R.; BALASTREIRE, L.A.; CAIXETA FILHO, J.V. Modelo de programação linear para seleção de pulverizadores agrícolas de barras. Engenharia Agrícola, Jaboticabal, v.24, n.2, p.355-63, 2004.

BATALHA, M. O. (Coord.) Gestão agroindustrial. São Paulo: Atlas, 1997. v.2. 324 p.

BULLOCK, D.G.; NIELSEN, R.L.; NYQUIST, W.E. A growth analysis comparison of corn grown in conventional and equidistant plant spacing. Crop Science, Madison, v.28, n.2, p.254-8, 1988.

ESPERANCINI, M.S.T.; PAES, A.R. Análise de investimentos da produção de café nos sistemas irrigado e convencional, na região de Botucatu, Estado de São Paulo. Informações Econômicas, São Paulo, v.5, n.34, p.52-60, 2005.

FETT, M.S. Análise econômica de sistemas de cultivo de macieiras no município de Vacaria-RS. 2000. 145 f. Dissertação. (Mestrado em Economia Rural) - Universidade Federal do Rio Grande do Sul, Porto Alegre, 2000.

FORSTHOFER, E.L.; SILVA, P.R.F. da. Desempenho agronômico e econômico do milho em diferentes níveis de manejo e épocas de semeadura. Pesquisa Agropecuária Brasileira, Brasília, v.41, n.3, p.399-407, 2006.

GALESNE, A.; FENSTERSEIFER, J.E.; LAMB, R. Decisões de investimento da empresa. São Paulo: Atlas, 1999. 295 p.

KREUZ, S. A.; SCHUCK, E.; PETRI, J.L. Avaliação econômica de alternativas de investimento no agronegócio da uva no meio oeste catarinense. Revista Brasileira de Fruticultura, Jaboticabal, v.27, n.2, p.230-7, 2005.

MIALHE, L. G. Manual de mecanização agrícola. São Paulo: Ceres, 1974. 301 p. 
OLIVEIRA, M.D.M.; MILAN, M. Ponto de renovação de tratores agrícolas de pneus: avaliação de uma frota. Agricultura em São Paulo, São Paulo, v.48, n.1, p.39-55, 2001.

REUNIÃO TÉCNICA ANUAL DE PESQUISA DE MILHO E SORGO (50 e 33: 2005: Porto Alegre). Indicações técnicas para cultivo de milho e sorgo no Rio Grande do Sul 2005-2006. Porto Alegre: FEPAGRO/Emater-RS/ASCAR, 2005. 155 p.

SANGÓI, L.; ARGENTA, G.; SILVA, P.R.F. da; MINETTO, T. J.; BISSOTO, V. Níveis de manejo na cultura do milho em dois ambientes contrastantes: análise técnico-econômica. Ciência Rural, Santa Maria, v.33, n.6, p.1.021-9, 2003.

SANGÓI, L.; ERNANI, P.R.; SILVA, P.R.F. da; HORN, D.; SCHMITT, A.; SCHWEITZER, C.; MOTTER, F. Rendimento de grãos e margem bruta de cultivares de milho com variabilidade genética contrastante em diferentes sistemas de manejo. Ciência Rural, Santa Maria, v.36, n.3, p.747-55, 2006.

SECRETARIA DA COORDENAÇÃO E PLANEJAMENTO. Atlas Socioeconômico do Rio Grande do Sul. On line. Disponível em: http:// /www.scp.rs.gov.br/ATLAS/default.asp. Acesso em: 15 dez. 2005.

SILVA, M.L. da; FONTES, A.A. Discussão sobre os critérios de avaliação econômica: Valor Presente Líquido (VPL), valor anual equivalente (VAE) e valor esperado da terra (VET). Revista Árvore, Viçosa, v.29, n.6, p.931-6, 2005.

SOFFNER, R.K.; MILAN, M.; RIPOLI, T.C.C. Gerenciamento global de sistema agrícola em unidades sucroalcooleiras através de programação linear. Stab - Açúcar, Álcool e Subprodutos, Piracicaba, v.11, n.5, p.16-19, 1993. 